\title{
DISTRIBUTION OF NEAREST NEIGHBORS IN DILUTE SUSPENSIONS OF MONODISPERSE SPHERES
}

\author{
Manuel ALONSO** \\ Department of Chemical Engineering, Osaka Prefecture University, \\ Sakai 593
}

Key Words: Random Packing, Limiting Packing Density, Nearest Neighbor Distribution, Rheology, Suspension Viscosity

\begin{abstract}
A model is proposed to describe the distribution of distances between nearest neighbors in random-packed low-density assemblies of equal-sized spheres. The model is based on the probability of not finding any sphere center in an arbitrary volume of a given size. This probability contains a limiting packing density, $\phi_{m}$, as a fitting parameter. The physical meaning of $\phi_{m}$ is the solids concentration above which every particle in the suspension is in contact with at least one of its neighbors. It means that at solids concentration $\phi=\phi_{m}$ the fraction of particles whose motions are constrained by the presence of any other contacting particles becomes unity. The value of $\phi_{m}$ is evaluated from nearest neighbor distributions in random assemblies of spheres obtained by computer simulation. The closeness of the resulting value, $\phi_{m}=0.52$, to that reported by Probstein et al. (1994) from viscosity measurements leads to the possibility of interpreting the empirical viscosity correlations in terms of the local arrangement of particles in the suspension.
\end{abstract}

\section{Introduction}

The packing of particles, whether in dry systems or in liquid suspensions, has been thoroughly studied through the years. The subject is important for the comprehension of any phenomena involving particulates, because the relative arrangement of particles affects the global behavior of the system. The flow of fluids through beds of granular materials (Dullien, 1979), the compaction of powders (Dickinson, et al., 1989), the segregation of components during solids mixing (Alonso, et al., 1991), and the rheology of suspensions (Ackerson, 1990) are instances of processes or phenomena for which understanding the particle packing properties is necessary.

The average solid volume fraction, $\phi$, is the parameter most frequently used to characterize the packing. However, when using $\phi$, a global property of the assembly, one is neglecting the possible effect of the local structure. The properties of two particulate assemblies having the same overall solid concentration (say $\phi=0.5236$ ) but different structure, one random-packed, the other simple cubic, will presumably be different, because the random assembly is isotropic whereas in the simple cubic structure there are preferential spatial directions. It would be desirable to characterize the local structure of particle packings in order to analyze its effect on the behavior of the system under consideration.

* Received on August 7, 1995.

** Dept. Materials Recycling, National Center of Metallurgical Research, CENIM-CSIC, Avenida Gregorio del Amo, 8, 28029 Madrid, Spain, Fax: +34 1-5347425
A model for the local void structure in twodimensional random packings of monodisperse disks was developed (Alonso, et al., 1992) and successfully applied to the study of segregation phenomena occurring during the diffusive mixing of solid particles (Alonso, et al., 1991). The model was later extended to polydisperse disks (Shinohara, et al., 1994b) and used to model the non-uniform permeation of fluids through particle beds ${ }^{11}$. The three-dimensional version of the void-size distribution model has also been developed (Alonso, et al., 1995) and shown to predict reasonably well experimental distributions measured in real beds.

These void-size distribution models were derived from knowledge of the probability $F(V, \phi)$ of not finding any particle center in a region of volume $V$ randomly selected within a packing of average solid volume fraction $\phi$. The evaluation of this probability goes back to Frenkel who treated the onedimensional case. However, the probability $F(V, \phi)$ as proposed by Frenkel, when extended to two or three dimensions, leads to void-size distributions which overpredict the experimental (or computer simulated) values, and a correction term has to be included. The correction, based on the existence of a certain fraction of void space which cannot be occupied by particles, was performed by introducing the density of the hexagonal close packing as a maximum or limiting density (Alonso, et al., 1995).

In the present study, the probability $F(V, \phi)$ is used to derive the distribution of distances between nearest neighbors in random assemblies of spherical particles of uniform size. The correction factor is not guessed a priori as in the void-size distribution model, 
but it is left as a fitting parameter to be determined from experimental results. The correction factor, $\phi_{m}$, has a very precise physical meaning: it represents the solid volume fraction above which every particle in the assembly is in contact with at least one of its neighbors. At concentrations greater than $\phi_{m}$ the motion of every particle is constricted by the presence of other contacting particle(s) in its neighborhood. This interpretation permits drawing a close analogy between the correction factor $\phi_{m}$ and a similar limiting density which appears as a fitting parameter, to be determine experimentally, in viscosity correlations for suspensions.

\section{Derivation of the Nearest Neighbor Distribution}

It has been shown (Alonso, et al., 1995) that the probability $F(V, \phi)$ of not finding any particle center in a region of volume $V$ arbitrarily selected within a random-packed assembly of uniform spheres is given by

$$
F(V, \phi)=k \exp \left[-\frac{\phi}{1-\frac{\phi}{\phi_{h c p}}}\left(\frac{V}{V_{p}}-1\right)\right]
$$

where $\phi$ is the average solid volume fraction in the assembly, $V_{p}$ the particle volume, $k$ a normalization constant, and $\phi_{h c p}=0.74$ the solid volume fraction of the hexagonal close packing structure. The latter was included into Eq. (1) as a limiting packing density to take into account the existence of void space not accessible to the particles. A void-size probability distribution was then derived from the basic Eq. (1) and found to be in good agreement with the distribution measured in a real bed of monodisperse spherical particles (Shinohara, et al., 1994a). It is important to point out that the correction factor $\phi_{h c p}$ affects only the $\phi$ appearing in the denominator of the exponential term in Eq. (1). At first sight it would seem reasonable that $\phi_{h c p}$ should also appear in the numerator of that term. This is not so because Eq. (1) can straightforwardly be derived from the fact that one has the certainty of finding a particle center when choosing an arbitrary volume of size $V_{c}(1-\phi)$ $\left.\phi_{h c p}\right)+V_{p}$, where $V_{c}$ is the volume of the container. It is only in this expression where $\phi_{h c p}$ enters as a correction factor (Alonso, et al., 1995).

The nearest neighbor distribution can easily be derived from Eq. (1) as follows. The probability $W(x, \phi)$ that the center-to-center distance between two nearest neighbors will be equal or greater than $x$ times the particle diameter is equal to the probability of not finding any particle center within the spherical shell of radii 1 and $x$ (see Fig. 1). Hence, $W(x, \phi)$ is equal to the function $F(V, \phi)$ evaluated for $V / V_{p}=\left[4 \pi\left(x^{3}-1\right) /\right.$

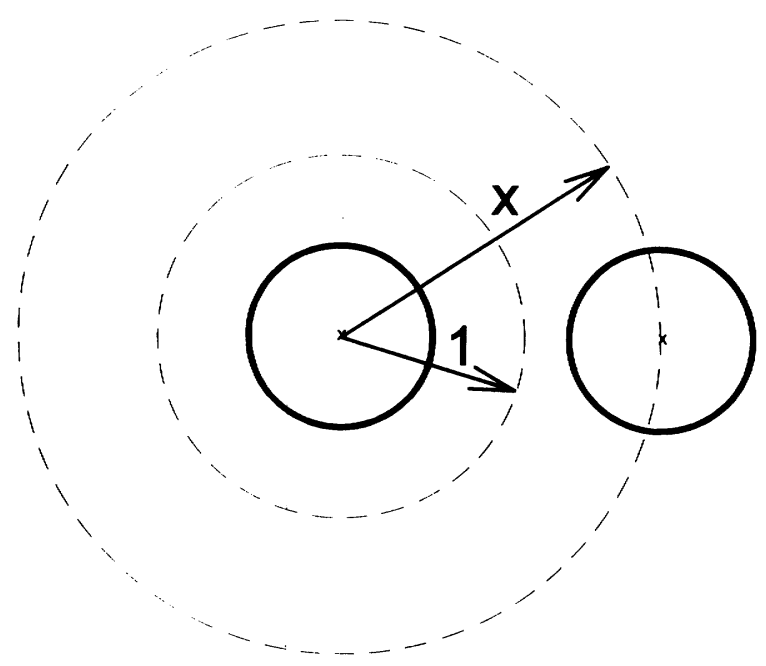

Fig. 1 Geometrical model for the evaluation of the nearest neighbor probability distribution

$3] /[\pi / 6]$. The normalization constant is evaluated by noting that the nearest neighbor distribution must satisfy the condition $W(1, \phi)=1$, which assures that the centers of any two nearest neighbors will be at least one particle diameter apart. Therefore, we have from Eq. (1)

$$
W(x, \phi)=\exp \left[-\frac{8 \phi\left(x^{3}-1\right)}{1-\frac{\phi}{\phi_{h c p}}}\right]
$$

However, Eq. (2) can not be correct, because it leads to the unreasonable result that each particle in the assembly will be, with probability one, in contact $(x=1)$ with at least one of its neighbors only when the packing has the maximum possible density $\phi_{\text {hcp }}$. On the contrary, it is well known that loose random packings have a density much lower, around 0.60 . Since these packings are obtained in the presence of gravity, we are sure that all the particles in them are in contact with at least one neighbor. Hence, one expects that the limiting state, in which there is at least one contact point per particle, will be achieved at densities much lower than 0.74 , and presumably lower than 0.60 .

Now, since $\phi_{h c p}$ appears in Eq. (2) as a correction factor, one can substitute it by another, yet undetermined, correction factor, say $\phi_{m}$, and write

$$
W(x, \phi)=\exp \left[-\frac{8 \phi\left(x^{3}-1\right)}{1-\frac{\phi}{\phi_{m}}}\right]
$$

To some extent, the introduction of the new correction factor is arbitrary, but it possesses a very precise 
physical meaning, for one finds from Eq. (3)

$$
W(x, \phi)=\left\{\begin{array}{l}
1 \text { for } x=1 \\
0 \text { for } x>1
\end{array}\right.
$$

Accordingly, $\phi_{m}$ is the critical solid volume fraction above which all the particles in the suspension are in contact with at least one neighbor. It can be also stated that above this concentration every particle in the assembly interacts with at least any other particle, or that the motion of all the particles in the suspension is restricted by the presence of contacting particle(s). At densities $\phi<\phi_{m}$ there is of course a certain fraction of particles whose motions are constrained by the presence of other particles in contact with them. What Eq. (4) says is that the fraction of constrained particles becomes unity at $\phi=\phi_{m}$. From these arguments it appears clear that there is an intimate connection between $\phi_{m}$ and the rheological behavior of the suspension, and this might prove to be useful in the reinterpretation of an analogous limiting density commonly used in viscosity correlations, as will be discussed below.

The probability density of nearest neighbors is obtained by differentiating Eq. (3),

$$
\begin{aligned}
w(x, \phi) \mathrm{d} x & =-\frac{\mathrm{d} W(x, \phi)}{\mathrm{d} x} \mathrm{~d} x \\
& =\frac{24 \phi x^{2}}{1-\phi / \phi_{m}} \exp \left[-\frac{8 \phi\left(x^{3}-1\right)}{1-\phi / \phi_{m}}\right] \mathrm{d} x
\end{aligned}
$$

$w(x, \phi) \mathrm{d} x$ is the probability that the center-to-center distance (measured as number of particle diameters) between two nearest neighbors is in the range between $x$ and $x+\mathrm{d} x$. Eq. (5) evaluated at $x=1$ gives the above mentioned fraction (probability density) of constrained particles as a function of the solids concentration.

The average distance between nearest neighbors can be evaluated as

$$
\begin{aligned}
\bar{x}(\phi) & =\int_{1}^{\infty} x w(x, \phi) \mathrm{d} x \\
& =1+\int_{1}^{\infty} W(x, \phi) \mathrm{d} x
\end{aligned}
$$

From Eqs. (4) and (6) one finds $\bar{x}\left(\phi_{m}\right)=1$ which means, of course, that in the limit $\phi=\phi_{m}$ any two nearest neighbors are in contact with one another, as was already clear from Eq. (4) itself.

\section{Evaluation of $\phi_{m}$ from Computer Simulations}

Nearest neighbor distributions in random assemblies of uniform spheres were generated by computer simulation as follows. A certain number (chosen to give the desired average solids concentration, $\phi$ ) of equal-sized spheres are randomly placed one by one in a box under the condition of no overlapping. The particle bed has periodic boundaries to prevent the presence of wall effects during packing formation, so that the assembly may be regarded as infinite along the three spatial directions. Once the packing of the desired density (reference unit) is formed, 26 identical packings (image units) are constructed around the reference unit by translation. The translation is done as follows. If the coordinates of the reference particle are $(\xi, \eta, \zeta)$, the coordinates of the image particles are $(\xi, \eta, \zeta+L),(\xi, \eta, \zeta-L),(\xi, \eta+$ $L, \zeta),(\xi, \eta-L, \zeta), \ldots$, where $L$ is the side length of the reference box. That is, the 27 points (one reference +26 images) are obtained from combinations of $[\zeta+(\mathrm{O},+L,-L)],[\eta+(0,+L,-L)]$ and $[\zeta+(0,+L,-L)]$. The 27 units altogether form a box whose side is three times the original. The computer program then searches for the closest neighbor to each one of the reference particles, and computes the distrubution function $W(x, \phi)$. A reference particle can have an image particle as its closest neighbor. This prevents obtaining unrealistically large values of $x$ for reference particles close to the wall.

A number of simulation runs were performed by varying (i) the average solid volume fraction (from 0.05 to 0.30 ), (ii) the size of the reference unit (from 8 to 20 particle diameters), and (iii) the (pseudo) random number generator seed. It was not possible to obtain random assemblies of density larger than about 0.32 using this simple simulation procedure. In order to achieve higher densities one should somehow compact the assembly to create, around the compacted packing, empty regions in which new particles could be accommodated. But in doing so, one would probably be introducing non-random feataures into the simulated packings. To avoid this, the present study has been restricted to dilute packings of maximum density 0.30 ; these packings can be strictly regarded as completely random.

From Eq. (3) and the total collection of $W(x, \phi)$ data from the simulation runs it was found $\phi_{m}=0.52$ as the best fitting value giving the minimum deviation between simulated and calculated probabilities. This does not mean that $\phi_{m}=0.52$ is necessarily the best fitting for each of the simulation runs: it is the best value to describe the totality of distributions obtained in this work. Figure 2 shows the simulated distributions along with those calculated by Eq. (3) using $\phi_{m}=0.52$. The data scattering at low densities, where most of the nearest neighbor distances are relatively large, is considerable. The goodness of the fitting improves for larger densities. From these data one should conclude that the limiting density is not a clear cut value but rather that, on the average, attains the value of 0.52 . 


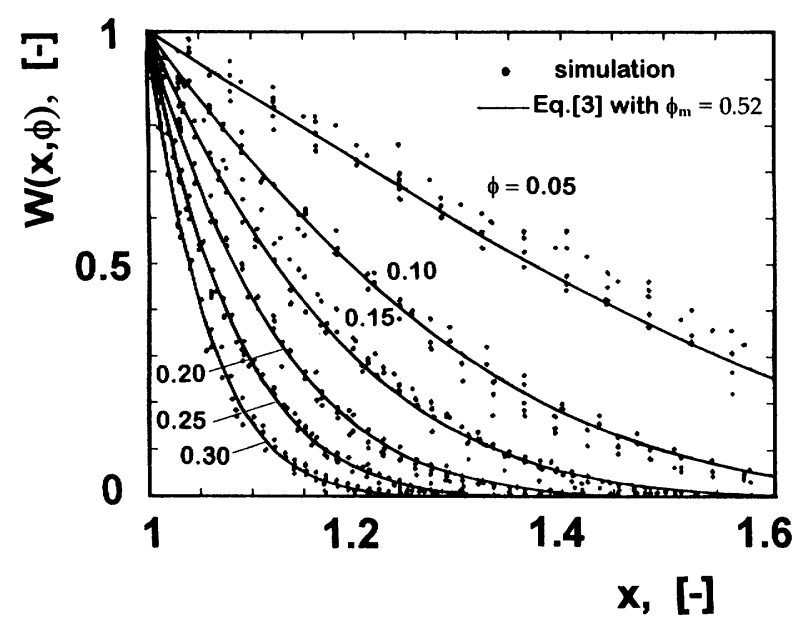

Fig. 2 Probability $W(x, \phi)$ of finding two nearest neighbors with centers separated a distance equal or larger than $x$ times the particle diameter, as a function of the solid volume fraction, $\phi$

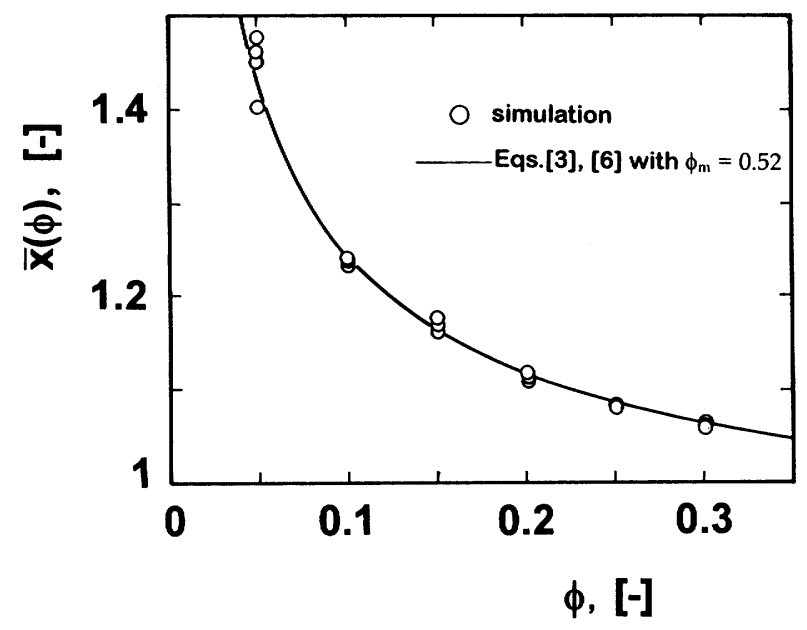

Fig. 3 Average center-to-center separation between nearest neighbors as a function of the solids concentration

The average distance between nearest neighbors, calculated numerically from Eq. (6), is plotted in Fig. 3. Except for $\phi=0.05$, the agreement is very good and proves that the basic model equation, Eq. (3), using $\phi_{m}=0.52$, describes properly the local packing structure of monodisperse spheres in terms of the distribution of distances between nearest neighbors.

It is worth mentioning that the value $\phi_{m}=0.52$ has been obtained for static assemblies of non interacting particles, i.e. in the absence of any force field whatsoever. This value should, in principle, be the same for flowing systems provided that the suspension does not present packing inhomogeneities.
3. Possible Connection between $\phi_{m}$ and the Limiting Density obtained from Viscosity Measurements

It is interesting to point out that the empirical correlations to evaluate the viscosity of a suspension as a function of the solids concentration contain also a maximum packing density as a fitting parameter to be determined experimentally (Chang, et al., 1994, Probstein, et al., 1994). The meaning of this parameter is that as the density of the suspension approaches the limiting value, the relative viscosity tends to infinity. To avoid confusion, the limiting density obtained from viscosity measurements will be denoted by $\phi_{m}^{\prime}$. There is a considerable scatter in the reported values of $\phi_{m}^{\prime}$, but recent viscosity measurements have yielded a value $\phi_{m}^{\prime}=0.524$ for monodisperse suspensions (Probstein, et al., 1994). Furthermore, they have shown that the numerical coincidence between this value and that of the packing density of the simple cubic structure is meaningless, because $\phi_{m}^{\prime}$ is actually obtained from the limiting random packing density reduced by a dilatancy factor of 1.19 , as confirmed by measurements in polydisperse suspensions.

The value of $\phi_{m}$ (correction term in the nearest neighbor distribution) obtained in the present work, 0.52 , is very close to that of $\phi_{m}^{\prime}$ obtained by Probstein et al. from viscosity measurements. Whether this is a mere coincidence or not is a point which should deserve further investigations. In particular, it should be desirable to extend the proposed approach to size-distributed systems to check whether the resulting value of $\phi_{m}$ is still coherent with those values of $\phi_{m}^{\prime}$ found from rheological experiments. However, the fact that $\phi_{m}$ can be thought as the density of the critical state in which there is at least one contact point per particle, is a strong reason to believe that, if not identical, $\phi_{m}$ and $\phi_{m}^{\prime}$ must be very intimately interrelated.

\section{Conclusions}

Nearest neighbor distributions in dilute suspensions of uniform spheres have been calculated by means of computer simulations. A semi-theoretical distribution has been derived which contains a correction factor as a fitting parameter. The correction factor, $\phi_{m}$, represents the critical solids concentration above which the motion of any particle in the suspension is constrained by the presence of a least one contacting particle. The value of $\phi_{m}$ obtained by fitting the simulated distributions, 0.52 , is very similar to the critical density obtained from viscosity meaurements. A possible connection between both critical densities has been proposed. 


\begin{tabular}{|c|c|c|}
\hline \multicolumn{3}{|c|}{ Nomenclatures } \\
\hline$F(V, \phi)$ & $=$ & $\begin{array}{l}\text { probability of not finding any particle in volume } V \\
\text { randomly chosen within random packing of density }\end{array}$ \\
\hline & & {$[-]$} \\
\hline$k$ & $=$ & normalization constant \\
\hline$V$ & $=$ & inspection volume chosen at random \\
\hline$V_{p}$ & $=$ & particle volume \\
\hline$V_{c}$ & $=$ & container volume \\
\hline$W(x, \phi)$ & $=$ & probability distribution of nearest neighbors $[-]$ \\
\hline$w(x, \phi)$ & $=$ & probability density of nearest neighbors \\
\hline$x$ & $=$ & $\begin{array}{l}\text { dimensionless distance between centres of nearest } \\
\text { neighbors }\end{array}$ \\
\hline $\bar{x}(\phi)$ & $=$ & $\begin{array}{l}\text { average dimensionless distance between nearest } \\
\text { neighbors }\end{array}$ \\
\hline$\phi$ & $=$ & average solid volume fraction \\
\hline$\phi_{\text {hcp }}$ & $=$ & density of hexagonal close packing \\
\hline$\phi_{m}$ & $=$ & critical packing density \\
\hline
\end{tabular}

Literature Cited

Ackerson, B. J.; J. Rheol., 34, 553-590 (1990).
Alonso, M., M. Satoh and K. Miyanami; Powder Technol., 68, 145152 (1991).

Alonso, M., M. Satoh and K. Miyanami; Can. J. Chem. Eng., 70, 2832 (1992).

Alonso, M., E. Sainz., F. A. Lopez and K. Shinohara; Chem. Eng. Sci., 50, 1983-1988 (1995).

Chang, C. and R. L. Powell; J. Rheol., 38, 85-98 (1994).

Dickinson, E., S. J. Milne and M. Patel; Powder Technol., 59, 11-24 (1989).

Dullien, F. A. L.; "Porous Media: Fluid Transport and Pore Structure," Academic Press, New York (1979).

Frenkel, J.; "Kinetic Theory of Liquids", Dover, New York, p. 127 (1955).

Probstein, R. F., M. Z. Sengun and T. C. Tseng; J. Rheol., 38, 811829 (1994).

Shinohara, K. and T. Murai; J. Ceram. Soc. Japan, 101, 1333-1338 (1994a).

Shinohara, K. and T. Murai; Kagaku Kogaku Ronbunshu, 20, 198204 (1994b). 